\title{
Clinical value of turbidity-elimination gout soup combined with external application of traditional chinese medicine to improve the pain and the volume of tophi in patients with gout
}

\author{
Hongbo LI ${ }^{1}$, Daqing NIE ${ }^{1}$, Shaoqiong WANG ${ }^{1}, \mathrm{Da} \mathrm{LI}^{2}$, Cheng LIU ${ }^{3 *}$
}

\begin{abstract}
To analyze the clinical value of turbidity-elimination gout soup combined with external application of traditional Chinese medicine to improve pain and the volume of tophi in patients with gout. A total of 94 patients with gout were selected as the study samples. They were randomly divided into control group treated with colchicine tablets and observation group treated with turbidity-elimination gout soup combined with external application of traditional Chinese medicine. Two groups of patients were compared before and after treatment in terms of pain, volume of tophi, score of TCM symptoms and laboratory indicators. There was no significant difference in NRS score, volume of tophi, redness and swelling of joints, heat and pain and other laboratory indicators between the two groups before treatment $(\mathrm{P}>0.05)$; NRS score, tophi, the integral of symptoms and other symptoms, and erythrocyte sedimentation rate, white blood cell count, blood uric acid level and etc. laboratory indexes of patients in the observation group were lower than those of the control group after treatment $(\mathrm{P}<0.05)$. Combination will effectively reduce the pain of patients with gout, reduce the volume of tophi, correct clinical symptoms and improve the level of laboratory indicators, with clinical reference and implementation efficiency.
\end{abstract}

Keywords: turbidity-elimination gout soup; external application of traditional Chinese medicine; gout; pain; volume of tophi.

Practical Application: The treatment of gout patients with turbidity-elimination gout soup combined with external application of traditional Chinese medicine will alleviate pain, reduce volume of tophi, improve clinical symptoms and correct the level of laboratory indicators in an effective way, serving as an effective treatment method with reference and implementation in clinical practice.

\section{Introduction}

Gout is a disease prevalent all over the world and may occur in all ages. The root cause of this disease is the excessive high level of blood uric acid, which causes deposition of uric acid crystals and thus triggers arthritis (Li et al., 2019a). Gout patients take joint redness, swelling, heat and pain as the typical symptoms. With the development of disease, the pain worsens gradually, which eventually leads to joint deformity and severely limited joint movement (Li et al., 2018). Studies have reported that gout may also lead to an increased risk of cardiovascular disease and endanger the lives of patients (Pérez Ruiz et al., 2019). Clinically, colchicine and other western drugs are often used to treat this disease, which can reduce the inflammatory response of patients and control the progress of disease (Angelidis et al., 2018) by inhibiting the action of white blood cells and reducing the formation of lactic acid. It is noted that colchicine is likely to cause adverse reactions in gastrointestinal tract, and water and electrolyte disorders may occur in severe cases. It is further pointed out in the study of Li et al. (2019b) that colchicine treatment may lead to an increased incidence of cataracts. For this reason, some scholars believe that traditional Chinese medicine may be used to avoid such problems, such as orally taking of traditional Chinese medicine, acupuncture, and external application of traditional Chinese medicine, aimed at removing blood stasis, clearing collaterals and removing gout, and many reports indicate that they have a good effect (You et al., 2019). However, until now, there have been relatively few studies on the combined application of traditional Chinese medicine in the treatment of gout. It is still unknown whether the combination of the two methods can help improve the treatment effect and whether it is safe and feasible. Based on this, it is proposed in this study the combination of turbidity-elimination gout soup and external application of traditional Chinese medicine, taking the gout patients admitted to our hospital as the objects to carry out the experiment, to explore the improvement effect of pain and volume of tophi by combining the two methods. The study is shown as following.

\section{Materials and methods}

\subsection{Data of objects}

A total of 94 patients with gout admitted to The Affiliated Hospital to Changchun University of Chinese Medicine from 
December 2018 till December 2019 were selected as study samples and divided into the control group and observation group by stratified random method, for 47 patients in each group respectively. The study was approved by The Affiliated Hospital to Changchun University of Chinese Medicine. Informed consent was obtained.

The control group was composed of 47 patients, with 31 men and 16 women; the age ranged from 24 to 69 years, with an average age of $(42.82 \pm 2.11)$ years; the course of disease ranged from 9 months to 20 years, with an average course of $(5.19 \pm 1.05)$ years; there were 152 203 tophi, and the average number of tophi was $(184.22 \pm 11.47)$. The observation group was composed of 47 patients, with 29 men and 18 women; the age ranged from 27 to 71 years, with an average age of (43.56 \pm 2.70) years; the course of disease ranged from 6 months to 18 years, with an average course of $(5.30 \pm 1.29)$ years; there were 149 205 tophi, and the average number of tophi was (180.67 \pm 12.05). The difference in the baseline data between the two groups was not statistically significant $(\mathrm{P}>0.05)$.

\subsection{Inclusion criteria}

1) All the patients met the diagnostic criteria for gout in Multidisciplinary Expert Consensus on the Diagnosis and Treatment of Hyperuricemia Related Diseases in China (Multi-Disciplinary Expert Task Force on Hyperuricemia and Its Related Diseases, 2017);2) The patients did not take non-steroidal anti-inflammatory drugs or hormones within 1 week before enrollment; 3) Patients are fully aware of the study protocol and voluntarily cooperate with drug therapy.

\subsection{Exclusion criteria}

1) Patients with severe organ dysfunction, such as heart, brain, lung, liver and kidney and etc.; 2) Patients with gastrointestinal ulcer; 3) The skin of affected part is damaged; 4) Stiff, deformed or incapacitated joints; 5) Those with mental illness and inability to communicate normally; 6) The control group was intolerant to colchicine; 7) The patients in the observation group were intolerant to turbidity-elimination gout soup and external application of traditional Chinese medicine; 8) Incomplete baseline data.

\subsection{Therapy}

The control group was treated with colchicine tablets (GYZZ H20113208) produced by Guangdong Bidi Pharmaceutical Co., Ltd. The initial dose was $1 \mathrm{mg} / \mathrm{per}$ time, 3 times/d, and the dose was changed to $0.5 \mathrm{mg} / \mathrm{per}$ time, 3 times $/ \mathrm{d}$ after $3 \mathrm{~d}$. The research group was treated with turbidity-elimination gout soup and external application of traditional Chinese medicine, and the prescription of turbidity-elimination gout soup: rhizoma smilacis glabrae $40 \mathrm{~g}$, yam rhizome $30 \mathrm{~g}$, semen coicis $30 \mathrm{~g}$, herba lycopi $15 \mathrm{~g}$, lumbricus $12 \mathrm{~g}$, red paeony root $12 \mathrm{~g}$, giant knotweed $10 \mathrm{~g}$, rhizoma alismatis $10 \mathrm{~g}$, radix clematidis $10 \mathrm{~g}$, peach kernel $10 \mathrm{~g}$, batryticated silkworm $10 \mathrm{~g}$ and liquorice $10 \mathrm{~g}$.

Based on the clinical symptoms of patients, $10 \mathrm{~g}$ of cortex phellodendron and $10 \mathrm{~g}$ of rhizoma anemarrhenae were added for severe heat and pain of the joint; for those with adverse joint flexion and extension, $10 \mathrm{~g}$ pangolin was added and $10 \mathrm{~g}$ the seed of cowherb was added; add $10 \mathrm{~g}$ safflower for blood stasis. Medicine preparation, 1 dose/d, divided in the morning and evening. External application: $50 \mathrm{~g}$ dried ginger, $50 \mathrm{~g}$ pantoginseng, $50 \mathrm{~g}$ ligusticum wallichii and $10 \mathrm{~g}$ borneol. After grinding into powder, mix with $50 \mathrm{~g}$ bran and $50 \mathrm{~mL}$ vinegar, heat until slightly dry, package in a linen bag and apply to the injured part, $20 \mathrm{~min} /$ per time, 1-2 times/d. Both groups were treated continuously for 21 days.

The diseased region of patients was examined through SOMATOM Definition 64 dual-source CT produced by Germany Siemens, the total radiation dose control in $4 \sim 10 \mathrm{mSV}$, scanning data transmission to the workstation, enter Gout software for analysis, based on the coefficient of material failure, urate crystals marked out green, the images were read by two hightitle physicians and the uric acid salt deposition was recorded.

The pain degree of patients was evaluated by digital evaluation scale (NRS), starting from 0 point and gradually transitioned to 10 points. Patients were marked in the table according to their subjective feelings, and the higher the score, the stronger the pain. The volume of tophi was calculated according to the results of dual-source CT.

The effect of two groups of patients with symptomatic improvement was evaluated with symptom integral of traditional Chinese medicine, the integral was prepared based on Guiding Principles for Clinical Research on New Traditional Chinese Medicine (Li et al., 2019c), including redness and swelling of joints, aggravation upon heat, adverse joint flexion and extension, scanty dark urine and constipation, evaluated as none -0 point, slight -2 points, medium - 4 points and severe -6 points.

Fasting peripheral blood of the patients was collected, centrifugation was performed, and serum was extracted. The Konelab 20 fullautomatic biochemical analyzer produced by Seymour Fisher was used to detect the erythrocyte sedimentation rate, white blood cell count, blood uric acid and other laboratory indicators.

\subsection{Statistical analysis}

The measurement unit data (NRS score, tophi volume, TCM symptom score and laboratory index) of the two groups of patients were recorded into SPSS17.0 for Windows for statistical analysis, which was expressed as mean \pm standard deviation, $t$ test, and $\mathrm{P}<0.05$ indicated that the difference between the groups was statistically significant.

\section{Results}

\subsection{Analysis of general data of patients in the two groups}

The results of inter-group comparison showed that the difference in baseline data between the two groups was not statistically significant $(\mathrm{P}>0.05)$, as shown in Table 1.

\subsection{Monitoring of treatment effect of typical cases}

Patient: male, 54 years, complained of right foot toe joint swelling and plantar thermal pain for many years, showing several 
Table 1. Comparison of Baseline Data of Patients in the Two Groups (n, $\%, \bar{x} \pm s)$.

\begin{tabular}{|c|c|c|c|c|c|c|}
\hline \multirow{2}{*}{ Group } & \multirow{2}{*}{ Case } & \multicolumn{2}{|c|}{ Gender } & \multirow{2}{*}{ Average Age (year) } & \multirow{2}{*}{$\begin{array}{c}\text { Average Course of } \\
\text { Disease (year) }\end{array}$} & \multirow{2}{*}{$\begin{array}{c}\text { Average Amount of } \\
\text { Tophi (pcs.) }\end{array}$} \\
\hline & & Male & Female & & & \\
\hline Observation group & 47 & $29(61.70 \%)$ & $18(38.30 \%)$ & $43.56 \pm 2.70$ & $5.30 \pm 1.29$ & $180.67 \pm 12.05$ \\
\hline Control group & 47 & $31(65.96 \%)$ & $16(34.04 \%)$ & $42.82 \pm 2.11$ & $5.19 \pm 1.05$ & $184.22 \pm 11.47$ \\
\hline$x^{2} / t$ & - & \multicolumn{2}{|c|}{0.393} & 1.481 & 0.453 & 1.463 \\
\hline $\mathrm{P}$ & - & \multicolumn{2}{|c|}{0.531} & 0.142 & 0.651 & 0.147 \\
\hline
\end{tabular}

Table 2. Changes of Pain and Tophi Volume in Observation Group and Control Group before and after Treatment $(\bar{x} \pm s)$.

\begin{tabular}{cccccccc}
\hline \multirow{2}{*}{ Group } & \multirow{2}{*}{ Case } & \multicolumn{2}{c}{ NRS score (point) } & & \multicolumn{2}{c}{ Volume of Tophi $\left(\mathrm{mm}^{3}\right)$} \\
\cline { 3 - 4 } & & Before Treatment & After Treatment & & Before Treatment & After Treatment \\
\hline Observation group & 47 & $6.57 \pm 0.81$ & $6.70 \pm 0.74$ & $1.22 \pm 0.34$ & & $20.85 \pm 1.44$ & $10.17 \pm 1.09$ \\
Control group & 47 & 0.812 & $3.83 \pm 0.60$ & & $20.52 \pm 1.76$ & $16.60 \pm 1.29$ \\
$t$ & - & 0.419 & 25.946 & 0.001 & 0.995 & 26.102 \\
$\mathrm{P}$ & - & & & & 0.322 & 0.001 \\
\hline
\end{tabular}

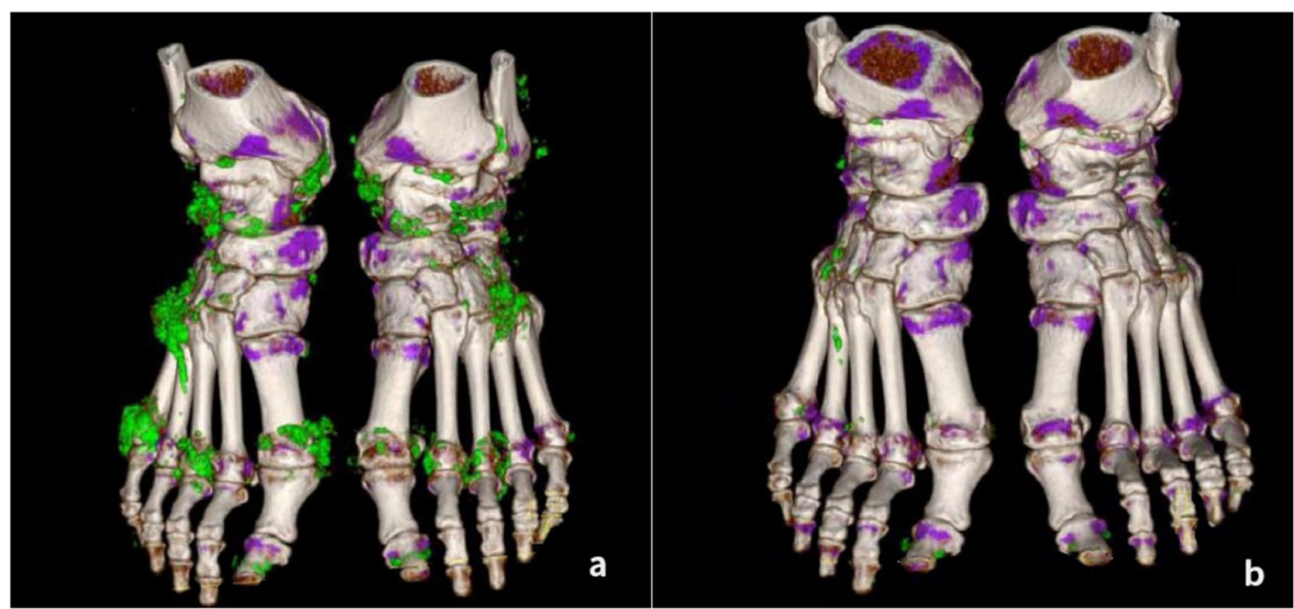

Figure 1. Monitoring of Treatment Effect of Typical Cases: (a) Dual-source CT Examination of Patient before Treatment; (b) Dual-source CT Examination of Patient after Treatment.

feet patchy green nodules through dual-source CT examination. The symptom control was good after being treated with turbidityelimination gout soup combined with external application of traditional Chinese medicine. The maximum area of urate was $3.3 \mathrm{~cm}^{2}$ before treatment and reduced to $1.65 \mathrm{~cm}^{2}$ after treatment, as shown in the Figure 1.

\subsection{Changes of pain and tophi volume in the two groups before and after treatment}

The results of inter-group comparison showed that there was no significant difference in NRS score and tophi volume between the two groups before treatment $(P>0.05)$. After treatment, the NRS score of the observation group was lower than that of the control group, and the volume of tophi was smaller, with statistically significant difference between the two groups $(\mathrm{P}<0.05)$, as shown in Table 2, Figure 2 and Figure 3.

\subsection{Changes of integral of traditional chinese medicine symptom in the two groups before and after treatment}

The comparison between the two groups showed that the difference in redness and swelling of joints, aggravation upon

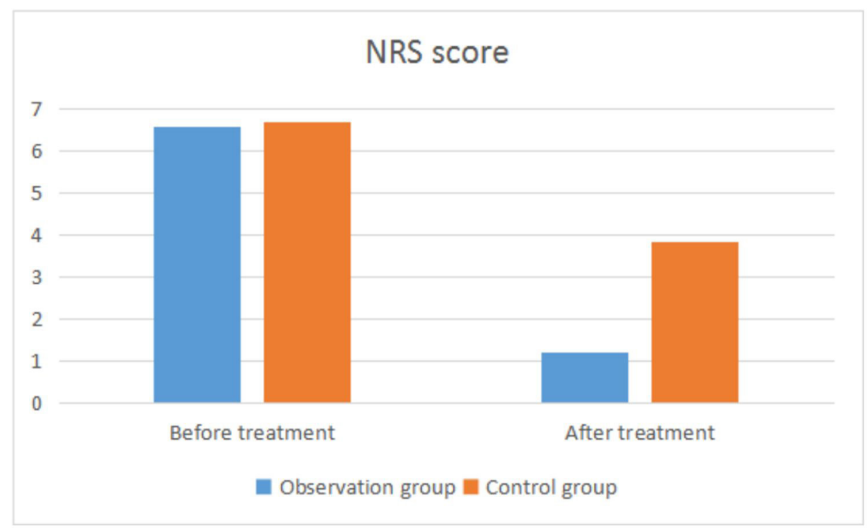

Figure 2. Changes of Pain in Observation Group and Control Group before and after Treatment.

heat, adverse joint flexion and extension, scanty dark urine and constipation and etc. was not statistically significant before treatment $(\mathrm{P}>0.05)$. Those in the observation group were lower than those in the control group after treatment, maintaining difference between groups with statistical significance $(\mathrm{P}<0.05)$, as shown in Table 3. 
Table 3. Changes of Integral of Traditional Chinese Medicine Symptom in the Two Groups before and after Treatment $(\bar{x} \pm s)$.

\begin{tabular}{|c|c|c|c|c|c|c|c|c|c|c|c|}
\hline \multirow{2}{*}{ Group } & \multirow{2}{*}{ Case } & \multicolumn{2}{|c|}{$\begin{array}{l}\text { Redness and Swelling } \\
\text { of Joints (points) }\end{array}$} & \multicolumn{2}{|c|}{$\begin{array}{l}\text { Aggravation upon Heat } \\
\text { (points) }\end{array}$} & \multicolumn{2}{|c|}{$\begin{array}{l}\text { Adverse Joint Flexion } \\
\text { and Extension (points) }\end{array}$} & \multicolumn{2}{|c|}{$\begin{array}{l}\text { Scanty Dark Urine } \\
\text { (points) }\end{array}$} & \multicolumn{2}{|c|}{ Constipation (points) } \\
\hline & & $\begin{array}{c}\text { Before } \\
\text { Treatment }\end{array}$ & $\begin{array}{c}\text { After } \\
\text { Treatment }\end{array}$ & $\begin{array}{l}\text { Before } \\
\text { Treatment }\end{array}$ & $\begin{array}{c}\text { After } \\
\text { Treatment }\end{array}$ & $\begin{array}{l}\text { Before } \\
\text { Treatment }\end{array}$ & $\begin{array}{c}\text { After } \\
\text { Treatment }\end{array}$ & $\begin{array}{l}\text { Before } \\
\text { Treatment }\end{array}$ & $\begin{array}{c}\text { After } \\
\text { Treatment }\end{array}$ & $\begin{array}{c}\text { Before } \\
\text { Treatment }\end{array}$ & $\begin{array}{c}\text { After } \\
\text { Treatment }\end{array}$ \\
\hline $\begin{array}{l}\text { Observation } \\
\text { group }\end{array}$ & 47 & $4.18 \pm 0.92$ & $1.19 \pm 0.26$ & $2.28 \pm 0.52$ & $0.71 \pm 0.13$ & $2.75 \pm 0.60$ & $0.76 \pm 0.17$ & $2.66 \pm 0.59$ & $0.68 \pm 0.17$ & $2.42 \pm 0.48$ & $0.57 \pm 0.17$ \\
\hline $\begin{array}{l}\text { Control } \\
\text { group }\end{array}$ & 47 & $4.05 \pm 0.88$ & $1.84 \pm 0.30$ & $2.31 \pm 0.49$ & $1.19 \pm 0.22$ & $2.77 \pm 0.53$ & $1.34 \pm 0.28$ & $2.72 \pm 0.56$ & $1.04 \pm 0.20$ & $2.46 \pm 0.44$ & $1.15 \pm 0.33$ \\
\hline$t$ & - & 0.700 & 11.225 & 0.288 & 12.878 & 0.171 & 12.139 & 0.506 & 9.403 & 0.421 & 10.712 \\
\hline $\mathrm{P}$ & - & 0.486 & 0.001 & 0.774 & 0.001 & 0.864 & 0.001 & 0.614 & 0.001 & 0.675 & 0.001 \\
\hline
\end{tabular}

Table 4. Changes of Laboratory Index in Observation Group and Control Group before and after Treatment $(\bar{x} \pm s)$.

\begin{tabular}{|c|c|c|c|c|c|c|c|}
\hline \multirow{2}{*}{ Group } & \multirow{2}{*}{ Case } & \multicolumn{2}{|c|}{ Erythrocyte Sedimentation Rate $(\mathrm{mm} / \mathrm{h})$} & \multicolumn{2}{|c|}{ White Blood Cell Count $\left(10^{9} / \mathrm{L}\right)$} & \multicolumn{2}{|c|}{ Blood Uric Acid $(\mu \mathrm{mol} / \mathrm{L})$} \\
\hline & & Before Treatment & After Treatment & Before Treatment & After Treatment & Before Treatment & After Treatment \\
\hline $\begin{array}{c}\text { Observation } \\
\text { group }\end{array}$ & 47 & $35.06 \pm 5.97$ & $19.20 \pm 6.22$ & $10.88 \pm 2.54$ & $4.63 \pm 0.85$ & $486.33 \pm 44.25$ & $366.94 \pm 32.69$ \\
\hline$t$ & - & 0.096 & 5.920 & 0.075 & 15.535 & 1.198 & 7.298 \\
\hline
\end{tabular}

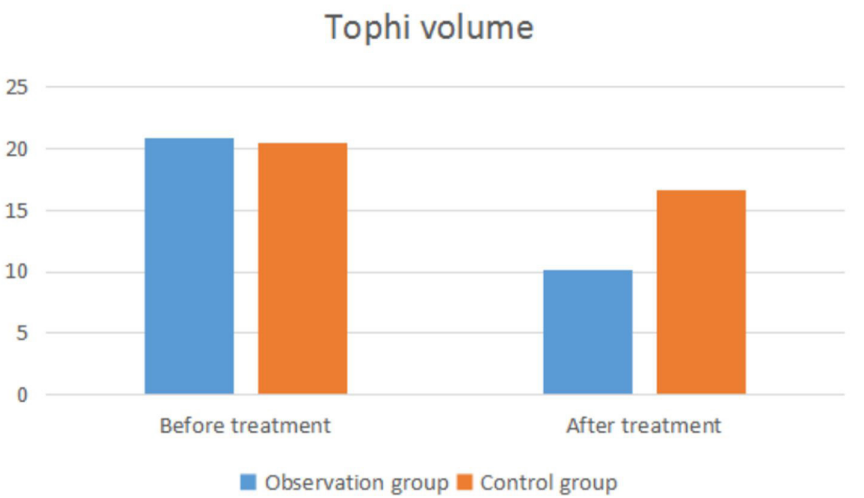

Figure 3. Changes of Tophi Volume in the Two Groups before and after Treatment.

\subsection{Changes of laboratory index in the two groups before and after treatment}

The comparison between the two groups showed that the difference in erythrocyte sedimentation rate, white blood cell count, blood uric acid level of laboratory indexes and etc. were not statistically significant before treatment $(\mathrm{P}>0.05)$. Those in the observation group were lower than those in the control group after treatment, maintaining difference between groups with statistical significance $(\mathrm{P}<0.05)$, as shown in Table 4 .

\section{Discussion}

Gout is a disease that may occur at any age, but mostly in men. Excessive blood uric acid level is the root cause of this disease, and the intake of high- purine food, use of drugs that inhibit uric acid excretion, excessive alcohol drinking, accompanied by diseases that lead to abnormal uric acid metabolism and etc. serve as the key factors leading to disorder of uric acid level
(Jeon et al., 2019). Gout patients are mainly manifested as joint redness, swelling, heat and pain, and some of them may have fever, chills, general malaise, tachycardia and other symptoms, which may even induce cardiovascular diseases, posing a serious threat to patients' quality of life and life safety (Lee et al., 2019).

Colchicine is a commonly used drug for treatment of gout. It is an alkaloid extracted from liliaceae drugs, which inhibits phagocytosis and activity of white blood cells to reduce uric acid crystal deposition and reduce pain response (Fu et al., 2017). It is noted that recent studies have suggested that this drug has no effect on patients with acute attacks and unsatisfactory therapeutic effect (Mu et al., 2019) on patients with younger age, higher body mass and higher blood uric acid level before treatment.

With the development and extension of TCM theory, more and more medical researchers tend to use TCM to treat gout. In traditional Chinese medicine theory, this disease belongs to the category of "calendaricity, arthralgia syndrome". Due to deficiency of body, deficiency of vital energy, combined with cold invasion or internal heat storage, patients may suffer from rheumatic fever arthralgia and arthralgia blocking meridians, resulting in blocked circulation of energy and blood flow and causing pains (Yu et al., 2018). Therefore, the treatment of this disease shall abide by the principle of clearing heat, removing rheumatism, benefiting humidity, and clearing collaterals. It is shown in the study of Huijuan et al. (2017) that the levels of blood uric acid and xanthine oxidase of rats suffering hyperuricemia treated with turbidity-elimination prescription are significantly improved, suggesting the feasibility of this method.

Based on the above theories, this study proposed the use of turbidity-elimination gout soup combined with traditional Chinese medicine therapy. In order to explore the effect of the combination of the two methods, this study carried out the experiment with 
the gout patients admitted to our hospital. The dual-source CT test results showed that the patients treated in this method had good symptom control effect and the maximum urate area was significantly reduced. Pain and tophi are the characteristics of patients with gout. The analysis of pain sensation and volume of tophi stone is an important criterion to evaluate the treatment effect of gout. The results showed that the NRS score of patients in the observation group was lower than that in the control group, and the volume of tophi was significantly reduced, suggesting that turbidity-elimination gout soup combined with traditional Chinese medicine external application therapy could effectively reduce the pain stress of patients and reduce the accumulation of urate crystals. Turbidity-elimination gout soup is composed of glabrous greenbrier rhizome, yam rhizome, coix seed, herba lycopi, lumbricus, root of common peony, polygonum cuspidatum, rhizoma alismatis, radix clematidis, peach kernel, batryticated silkworm, licorice and other ingredients, and cortex phellodendri, rhizoma anemarrhenae, pangolin scales, cowherb seed, safflower and other medicine may be added or reduced based on the state of illness. Glabrous greenbrier rhizome, yam rhizome and radix clematidis are monarch drugs. Glabrous greenbrier rhizome is used for detumescence, detoxification and benefiting humidity; yam rhizome is used for benefiting humidity, eliminating rheumatism and dispelling pain; radix clematidis is used for eliminating rheumatism, dispelling pain, dehumidification and dredging collaterals (Wang et al., 2019). The coix seed, herba lycopi, lumbricus, root of common peony, polygonum cuspidatum, rhizoma alismatis, radix clematidis, peach kernel, batryticated silkworm are adjuvant drugs. Coix seed is used for tonifying spleen, clearing damp and inducing diuresis to alleviate edema; herba lycopi is used for promoting circulation and removing stasis, diuresis and detumescence; lumbricus is used for dredging collaterals and diuresis; root of common peony is used for removing heat to cool blood; polygonum cuspidatum and peach kernel are used for removing stasis and dredging collaterals; radix clematidis is used for purging heat and excreting dampness; batryticated silkworm is used for eliminating rheumatism and relieving pain (Nam et al., 2017). The entire prescription has the effect of removing rheumatism and clearing collaterals, relieving muscle and relieving pain. On this basis, dried ginger, panax notoginseng, ligusticum chuanxiong, borneol and other drugs will be added for external application. The dry ginger is used for warming and dispersion of coldness; panax notoginseng and ligusticum chuanxiong will promote blood circulation and relieve pain; borneol shall clear heat and disperse poison, and heat compress after adding bran and vinegar can also promote blood circulation in the affected part, relieve pain and shrunken tophi (Chen \& Chen, 2019).

Further attention was paid to the effect and principle of symptom improvement by combining the two methods, and TCM syndrome score was selected as the main evaluation criterion. It's pointed out in the study that the symptom score of the observation group was lower than that of the control group, including joint redness, heat and pain, aggravated by heat, adverse joint flexion and extension, shortness of urination, and defecation and etc. It's likely to be related to the addition and reduction of different drugs, such as such as cortex phellodendri, anemarrhena, pangolin, kingsbane, safflower and other drugs, based on the specific symptoms of the patients. Such kind of medicine belongs to adjuvant in the prescription, among which golden cypress decreases internal heat and eliminates dampness; Anemarrhensis can relieve heat from fire; Pangolin can eliminate carbuncle and promote blood circulation; the seed of cowherb can diuretic tong drench; Safflower can disperse dampness and remove stasis (Yan et al., 2018). Specific drugs were added and subtracted to improve the TCM symptom score significantly based on the different clinical manifestations of patients.

Erythrocyte sedimentation rate is the rate at which red blood cells naturally sink in the blood and is closely related to erythrocyte and plasma factors. When immune diseases, inflammatory diseases, hypercholesterolemia, gout appear, erythrocyte sedimentation rate will increase significantly; White blood cell count is a basic indicator in routine blood tests, which can be used for the diagnosis and differentiation of various diseases. At present, it is believed that patients with gout can also be diagnosed by white blood cell count. When the disease attacks, the index will appear pathological increase; Blood uric acid is the product of the re-oxidation of purines in the liver and the abnormally high index is a key cause of gout. The observation of the above laboratory indicators is the "gold standard" for evaluating the therapeutic effect of patients. The results showed that the erythrocyte sedimentation rate, white blood cell count, uric acid and other laboratory indicators in the observation group were lower than those in the control group, which indicated that the treatment through turbidity-elimination gout soup combined with external application of traditional Chinese medicine wass likely to inhibit gout progression by correcting the patients' biochemical indicators, which might be related to the pharmacological effects of drugs used in gout decoction, for example, rhizoma smilacis glabrae could control the oxidation of purine and reduce uric acid secretion by inhibiting the activity of xanthine oxidase; Coix seed could reduce the blood uric acid level by lowering the conversion rate of uric acid precursor; Knotweed could control gout symptoms through the TLRs/My D88 pathway, thereby regulating erythrocyte sedimentation rate and white blood cell count.

To sum up, the treatment of gout patients with turbidityelimination gout soup combined with external application of traditional Chinese medicine will alleviate pain, reduce volume of tophi, improve clinical symptoms and correct the level of laboratory indicators in an effective way, serving as an effective treatment method with reference and implementation in clinical practice.

\section{References}

Angelidis, C., Kotsialou, Z., Kossyvakis, C., Vrettou, A. R., Zacharoulis, A., Kolokathis, F., Kekeris, V., \& Giannopoulos, G. (2018). Colchicine pharmacokinetics and mechanism of action. Current Pharmaceutical Design, 24(6), 659-663. http://dx.doi.org/10.2174/13816128246661 80123110042. PMid:29359661.

Chen, Y., \& Chen, X. (2019). Ancient herbal component may be a novel therapeutic for gouty arthritis. Journal of Leukocyte Biology, 105(1), 7-9. http://dx.doi.org/10.1002/JLB.3CE1018-384R. PMid:30517770.

Fu, T., Cao, H., Yin, R., Zhang, L., Zhang, Q., Li, L., \& Gu, Z. (2017). Associated factors with functional disability and health-related 
quality of life in Chinese patients with gout: a case-control study. BMC Musculoskeletal Disorders, 18(1), 429. http://dx.doi.org/10.1186/ s12891-017-1787-7. PMid:29100504.

Huijuan, W., Xiaoxu, C., Rui, S., Xinghui, L., Beibei, T., \& Jianchun, M. (2017). Qi-Zhu-Xie-Zhuo-Fang reduces serum uric acid levels and ameliorates renal fibrosis in hyperuricemic nephropathy rats. Biomedicine and Pharmacotherapy, 91, 358-365. http://dx.doi. org/10.1016/j.biopha.2017.04.031. PMid:28463799.

Jeon, Y. S., Hwang, D. S., Hwang, J. M., Lee, J. K., \& Park, Y. C. (2019). Pathological fracture of the femoral neck due to tophaceous gout: an unusual case of Gout. Hip \& Pelvis, 31(4), 238-241. http://dx.doi. org/10.5371/hp.2019.31.4.238. PMid:31824879.

Lee, S. Y., Park, W., Suh, Y. J., Lim, M. J., Kwon, S. R., Lee, J. H., Joo, Y. B., Oh, Y. K., \& Jung, K. H. (2019). Association of serum uric acid with cardiovascular disease risk scores in Koreans. International Journal of Environmental Research and Public Health, 16(23), E4632. http:// dx.doi.org/10.3390/ijerph16234632. PMid:31766442.

Li, Q. H., Liang, J. J., Chen, L. X., Mo, Y. Q., Wei, X. N., Zheng, D. H., \& Dai, L. (2018). Clinical characteristics and renal uric acid excretion in early-onset gout patients. Zhonghua Nei Ke Za Zhi, 57(3), 185190. PMid:29518862.

Li, Q., Li, X., Wang, J., Liu, H., Kwong, J. S., Chen, H., Li, L., Chung, S. C., Shah, A., Chen, Y., An, Z., Sun, X., Hemingway, H., Tian, H., \& Li, S. (2019a). Diagnosis and treatment for hyperuricemia and gout: a systematic review of clinical practice guidelines and consensus statements. BMJ Open, 9(8), e026677. http://dx.doi.org/10.1136/ bmjopen-2018-026677. PMid:31446403.

Li, Y. J., Perng, W. T., Tseng, K. Y., Wang, Y. H., \& Wei, J. C. (2019b). Association of gout medications and risk of cataract: a populationbased case-control study. QJM, 112(11), 841-846. http://dx.doi. org/10.1093/qjmed/hcz167. PMid:31286139.

Li, Y., Piranavan, P., Sundaresan, D., \& Yood, R. (2019c). Clinical characteristics of early-onset gout in outpatient setting. ACR Open Rheumatology, 1(7), 397-402. http://dx.doi.org/10.1002/acr2.11057. PMid:31777819.

Mu, Z., Wang, W., Wang, J., Lv, W., Chen, Y., Wang, F., Yu, X., Wang, Y., Cheng, B., \& Wang, Z. (2019). Predictors of poor response to uratelowering therapy in patients with gout and hyperuricemia: a post-hoc analysis of a multicenter randomized trial. Clinical Rheumatology,
38(12), 3511-3519. http://dx.doi.org/10.1007/s10067-019-04737-5. PMid:31410659.

Multi-Disciplinary Expert Task Force on Hyperuricemia and Its Related Diseases. (2017). Chinese multi-disciplinary consensus on the diagnosis and treatment of hyperuricemia and its related diseases. Zhonghua Nei Ke Za Zhi, 56(3), 235-248. PMid:28253612.

Nam, J. S., Jagga, S., Sharma, A. R., Lee, J. H., Park, J. B., Jung, J. S., \& Lee, S. S. (2017). Anti-inflammatory effects of traditional mixed extract of medicinal herbs (MEMH) on monosodium urate crystal-induced gouty arthritis. Chinese Journal of Natural Medicines, 15(8), 561-575. http://dx.doi.org/10.1016/S1875-5364(17)30084-5. PMid:28939019.

Pérez Ruiz, F., Richette, P., Stack, A. G., Karra Gurunath, R., García de Yébenes, M. J., \& Carmona, L. (2019). Failure to reach uric acid target of $<0.36 \mathrm{mmol} / \mathrm{L}$ in hyperuricaemia of gout is associated with elevated total and cardiovascular mortality. RMD Open, 5(2), e001015. http://dx.doi.org/10.1136/rmdopen-2019-001015. PMid:31673414.

Wang, S., Fang, Y., Yu, X., Guo, L., Zhang, X., \& Xia, D. (2019). The flavonoid-rich fraction from rhizomes of Smilax glabra Roxb. ameliorates renal oxidative stress and inflammation in uric acid nephropathy rats through promoting uric acid excretion. Biomedicine and Pharmacotherapy, 111, 162-168. http://dx.doi.org/10.1016/j. biopha.2018.12.050. PMid:30579255.

Yan, Y. Q., Sun, J., Zhang, L., Miao, L. L., \& Xie, Z. J. (2018). Analysis on mechanisms and medication rules of herbal prescriptions for gout caused by heat-damp accumulation syndrome based on data mining and network pharmacology. Zhongguo Zhongyao Zazhi, 43(13), 2824-2830. PMid:30111037.

You, W., Wang, J., Zou, Y., Che, K., Hou, X., Fei, H., \& Wang, Y. (2019). Modified Chuanhu anti-gout mixture, a traditional Chinese medicine, protects against potassium oxonate-induced hyperuricemia and renal dysfunction in mice. The Journal of International Medical Research, 47(5), 1927-1935. http://dx.doi.org/10.1177/0300060519831182. PMid:30832523.

Yu, X. N., Wu, H. Y., Deng, Y. P., Zhuang, G. T., Tan, B. H., Huang, Y. Z., Tang, S. Y., Tu, X., Jordan, J. B., \& Zhong, S. (2018). "Yellow-dragon Wonderful-seed Formula" for hyperuricemia in gout patients with dampness-heat pouring downward pattern: a pilot randomized controlled trial. Trials, 19(1), 551. http://dx.doi.org/10.1186/s13063018-2917-8. PMid:30314508. 